\title{
Cocteau et la poétique du déplacement
}

Réflexions sur la genèse de Morceaux choisis (1932)

\section{François Rouget}

\section{(2) OpenEdition \\ 1 Journals}

\section{Édition électronique}

URL : http://journals.openedition.org/genesis/561

DOI : 10.4000/genesis.561

ISSN : 2268-1590

Éditeur :

Presses universitaires de Paris Sorbonne (PUPS), Société internationale de génétique artistique littéraire et scientifique (SIGALES)

\section{Édition imprimée}

Date de publication : 1 janvier 2011

Pagination : 205-218

ISBN : 978-2-84050-749-9

ISSN : 1167-5101

\section{Référence électronique}

François Rouget, "Cocteau et la poétique du déplacement », Genesis [En ligne], 32 | 2011, mis en ligne le 18 septembre 2012, consulté le 20 avril 2019. URL : http://journals.openedition.org/genesis/561 ; DOI : 10.4000/genesis.561 


\title{
Cocteau et la poétique du déplacement
}

Réflexions sur la genèse de Morceaux choisis (1932)

\author{
François Rouget
}

$\mathrm{L}$ 'étude de la poésie nous apprend que l'un des premiers gestes du poète consiste à conjurer sa hantise de la dissémination par le rassemblement des poèmes en recueil. Dans ce passage du texte au livre, le poème acquiert une nouvelle physionomie et ébauche avec les autres pièces une relation nouvelle1. L'économie ainsi constituée du livre varie en fonction du type de recueil : volume, collection, série, album, anthologie ou florilège. Réalisé par l'auteur ou par une autre personne - un proche tel un parent, un ami ou l'éditeur -, le recueil résulte d'un effort concerté pour donner unité au disparate et présenter au lecteur des poèmes dans un contexte nouveau. En changeant l'espace et le temps qui avaient présidé à l'apparition du poème, l'écrivain invite le critique à questionner les mobiles, les enjeux et les conséquences de son geste.

Cette démarche prend une tournure esthétique singulière - et même dramatique - dans le cas de Jean Cocteau qui, peut-être plus que tout autre poète français du $x^{e}$ siècle, n'a cessé de s'interroger sur le sens du poème et l'unité du recueil. À l'instar de Ronsard qui avait multiplié les éditions de ses Euvres pour intégrer ses pièces séparées et atteindre un équilibre incertain, Cocteau a réédité et/ou rassemblé les recueils épars de ses poésies princeps, puis les a fait évoluer en déplaçant, supprimant, ajoutant et corrigeant des textes dont l'histoire génétique est aussi complexe que passionnante 2 . Ce sont précisément les modalités des déplacements opérés par le poète dans son œuvre que nous souhaitons étudier à partir de l'exemple de Morceaux choisis ${ }^{3}$, auto-anthologie, album ou florilège de poèmes composés et publiés de 1916 à 19274. À partir de l'enquête génétique de ce recueil, qui s'appuie sur l'étude des éditions séparées et collectives antérieures des poèmes qui le composent, il s'agira de s'interroger sur le statut de ce livre délaissé de la critique, sur les objectifs de l'écrivain, et sur les effets esthétiques (plastiques, musicaux) produits par le déplacement et le nouvel agencement des poèmes. On verra que Cocteau compose un nouveau livre pour reconfigurer ses recueils antérieurs et ébaucher une unité provisoire de ses poésies qui furent louées ou détestées de son public. Au moment où il tente de conjurer ses démons (l'attrait de l'opium, une sexualité contrariée, l'angoisse de la mort), Cocteau procède à un bilan personnel et poétique des vingt années précédentes, et entend refonder les bases de sa vision poétique qui doivent lui permettre de dépasser ses échecs.

Le livre de Morceaux choisis signale une double attitude de repli sur soi et de reconquête du monde. L'espace du livre fait entrer le lecteur dans l'intimité voilée de l'homme blessé mais surtout dans l'atelier du poète qu'il observe au travail. La poésie fait alors l'objet de multiples remaniements et d'expérimentations qui seront repris par la suite lors de la réédition des Poésies en 1947 et 1956.

\section{Morceaux choisis, une anthologie restreinte}

On ne connaît pas les circonstances qui ont présidé à la publication de Morceaux choisis et les fonds d'archives relatifs à la vie et à l'œuvre de Jean Cocteau n'apportent

1. Sur cette poétique du recueil, voir les études rassemblées par D. Alexandre, M. Frédéric et J.-M. Gleize, dans Méthode !, n 2, «Le Recueil poétique », 2002.

2. Voir l'édition des CEuvres poétiques complètes de Jean Cocteau, éd. M. Décaudin, Paris, Gallimard, coll. «Bibliothèque de la Pléiade », 2005 [1re éd. 1999], p. LVI.

3. Morceaux choisis. Poèmes, Paris, Gallimard, 1932 ( $5^{e}$ édition»). Toutes nos références, sauf mention contraire, renvoient à cette édition.

4. Le volume rassemble une sélection de poèmes publiés précédemment dans Le Cap de Bonne-Espérance (1916-1919), Discours du grand sommeil (1916-1918), Poésies (1920), L'Ode à Picasso (1925), Vocabulaire (1922), Plain-chant (1923) et Opéra (1925-1927). 
aucune lumière sur le motif de cette publication. À la date de 1932, Cocteau a déjà publié des pièces de théâtre, des romans bien accueillis, et réuni l'essentiel de son œuvre poétique dans des recueils augmentés au cours des rééditions (Poésies [1917-1920] en 1920, Poésies 1916-1923 en 1925, 1936, 1947 et 1956). S'il continue à publier des livres inédits de poésie à intervalles réguliers, il s'attache dans le même temps à les intégrer au bloc existant depuis 1925 .

Dans ces conditions, on peut s'interroger sur les raisons qui poussent le poète à procurer au public en juin 1932 non pas une édition augmentée de sa poésie mais au contraire un florilège restreint, tel un album de fleurs choisies par le poète, et peut-être à la demande de son éditeur Gaston Gallimard. Peu après la parution d'Opium (ou « journal d'une désintoxication », 1930), et simultanément à celle d'Essai de critique indirecte, c'est en lecteur de son œuvre que Cocteau produit son nouveau livre5. À l'exemple des Morceaux choisis de Claudel, parus à la NRF en 1925, le livre de Cocteau recueille des pièces antérieures qui délimitent un espace de recueillement propice au resserrement fécond. Car s'il accueille quelques inédits, le volume des Morceaux choisis opère une sélection, des coupes sombres dans les recueils antérieurs, et ne retient que ce qui correspond au tempérament du poète en 1931 , à ses préférences. Le livre prend la forme d'un bilan personnel et poétique, non point pour constituer un « jardin de plaisance », ou un album poétique réunissant les compositions de jeunesse sur lesquelles l'écrivain porterait un regard attendri et nostalgique, mais pour s'interroger avec lucidité sur le chemin accompli et porter un regard critique sur les formes expressives explorées jusquelà. En s'écartant provisoirement de la trajectoire accumulative qui consiste à bâtir l'unité de l'œuvre, Cocteau déplace sa poésie dans une voie adjacente, la scrute, en retire les éléments les plus significatifs ou les plus beaux de son point de vue, pour les présenter au public sous un nouveau jour, sous un éclairage restreint et signifiant.

À vrai dire, le titre général, de nature « rhématique 6 », qui domine la page de titre suggère la modestie de l'ambition de son auteur qui a sélectionné des éléments de sa production antérieure. Le sous-titre «Poèmes » en définit simplement le contenu. En somme, le livre n'offre rien de nouveau et se tourne vers le passé, signe peut-être que le poète est parvenu à la fin d'un cycle, ou de sa vie. Mais l'indication de "cinquième édition », toujours en page de titre, semble neutraliser cet effet nostalgique. Cocteau, qui prête un soin minutieux à la datation de ses œuvres (composition et impression), signale un classement et donc un processus. Le caractère désinvolte ou génériquement indéfini du titre se voit contredit par une précision qui relève de la génétique. La page de titre présente les indices du projet de l'écrivain qui a choisi de porter son attention sur un échantillon de poèmes pour en offrir un cinquième état, provisoire (sinon il aurait précisé qu'il s'agissait de l'« édition définitive »). En somme, le nouveau livre ne correspond nullement à un livre au rabais, ou un hommage composé de «best of» d'un poète disparu, ou encore d'un florilège élaboré par l'éditeur pour satisfaire la curiosité du public. Il se pose comme un livre-bilan, situé dans une dialectique de l'ancien et du nouveau, ou encore une halte effectuée par le poète pour mieux repartir.

La présence ostentatoire de Cocteau par un autoportrait placé au seuil de son livre suggère clairement l'interpellation du lecteur par le poète. Dessiné de demi-profil, en buste, Cocteau semble tourner ses regards vers la page de départ (faux-titre) et scruter avec nervosité la réaction du lecteur. De plus, à nouvel ouvrage, nouvelle préface, qui ne sera plus jamais réimprimée dans les œuvres de Cocteau, ce qui en fait un document original7 ${ }^{7}$, et d'autant plus intéressant que le poète de son aveu « répugne aux préfaces 8 ». Cocteau

5. Sur l'évocation de la vie et la carrière de Cocteau durant ces années 1930-1932, on consultera la «Chronologie » figurant en tête de l'édition de la «Bibliothèque de la Pléiade », op. cit., p. XLI-XLII, et la biographie de l'écrivain par C. Arnaud, Jean Cocteau, Paris, Gallimard, 2003, p. 439 $s q$.

6. Voir G. Genette, Seuils, Paris, Éditions du Seuil, 1987, en particulier les pages 82,89 et 95 .

7. Ce texte est absent du volume des Euvres poétiques complètes dans la «Bibliothèque de la Pléiade », régi par le principe d'édition chronologique, qui n'accorde aucune attention spécifique à Morceaux choisis. 8. Comme il le confie paradoxalement dans la préface du Cap de BonneEspérance en 1918 ; voir l'édition citée, p. 6, et la notice correspondante, p. 1571. 
compose à cette occasion un texte préliminaire bref, métaphorique, qui se double d'un programme littéraire :

Il existe des poèmes où le poète essaye sa chance; d'autres où le poète la prolonge. Rares sont les poèmes de chance. Ils coulent de la main comme l'ectoplasme de la bouche du médium. Le poète, endormi d'un œil, contrôle la descente.

Ainsi naissent des mandragores : statues des profondeurs. Réunir cette sorte de poèmes, parfois rébarbatifs, les couper, les consolider, impose une tâche ingrate ; car le public aime reconnaître, la connaissance le fatigue et rarement il approuve ce que le poème apporte de nouveau.

Ce préambule métapoétique ou court poème en prose a fait l'objet d'une lente maturation, comme l'atteste le jeu des premières épreuves corrigées de la main de Cocteau, document inconnu à ce jour et qui apporte un éclairage singulier sur son projet éditorial et poétique ${ }^{9}$ (voir fig. 1). Modifié à plusieurs reprises, ce texte définit dans un premier temps une conception de la poésie à la fois inspirée (nouvelle et étonnante) et élaborée par l'artisan des lettres (la production du passé), puis il commente l'effort pour recueillir et retoucher les poèmes. La fin de cette préface souligne le désenchantement d'un écrivain face à un public passif qui n'attend rien de nouveau. Celui-ci s'attend donc à relire dans Morceaux choisis un assortiment de pièces anciennes. Or Cocteau paraît s'être ingénié à remodeler de fond en comble sa production antérieure pour lui conférer une physionomie nouvelle dans laquelle le lecteur garde ses repères et peut « reconnaître » une matière antérieure, apprivoisée.

Le statut de cette « préface » n'a rien de durable, à l'image de tous ces poèmes qui, une fois encore, sont retouchés par le poète. Ses fonctions sont diverses et posent plusieurs questions relatives au destinataire (avoué ou réel) et aux raisons de sa composition. Au seuil du recueil qui préfigure la lecture, à qui s'adresse-t-elle ? Sans doute au lecteur de 1932 à qui Cocteau présente une nouvelle mouture de son œuvre poétique, mais aussi au lecteur posthume, car le public de 1932 qui renâcle à la surprise ne peut apprécier la démarche si sinueuse d'un artiste protéiforme. Pourquoi Cocteau choisit-il de l'écrire alors que ses poèmes ne méritent plus d'être présentés ? Sans doute pour en suggérer l'unité formelle ou thématique, pour les « réunir », les « consolider » dans une nouvelle structure exogène. Mais au-delà de l'unité envisagée, Cocteau s'efforce ici de définir la genèse de l'écriture en la rattachant à la conception platonicienne de l'inspiration, de la «fureur » ou force transmise des Muses qui se voit canalisée par « la bouche du médium » ou la main du poète inspiré, selon une conception emblématique du poète génial qui parcourt la littérature de Pindare à Saint-John Perse, et qui se voit développée et illustrée tout au long des Morceaux choisis. La «préface » de ce recueil donne ainsi l'occasion à Cocteau d'énoncer un art poétique qui définit la poésie comme une exploration incessante, une activité spirituelle magique, et présente le poète comme le récepteur d'une grâce qu'il lui faut contrôler. C'est à l'aune de ce court poème métatextuel que Cocteau défend son œuvre et invite à la percevoir sous un jour nouveau.

De fait, cette sélection inédite des « Poèmes » est loin de se réduire à un florilège sommaire. Contrairement à ce que laisserait croire la table des matières - qui énumère simplement dans l'ordre chronologique de parution les titres des recueils dont sont extraits les poèmes reproduits ici, obligeant le lecteur à parcourir le livre pour identifier les pièces retenues -, le contenu de Morceaux choisis révèle une élaboration soignée. Si l'on retrouve tous les thèmes favoris de Cocteau (la guerre, la mort, les paradis artificiels, l'amour, le rêve), on y observe cependant une mise en valeur de l'image du poète et de sa mission. Le resserrement de l'œuvre a conduit le discours à l'autoréflexivité. Il ressort de la lecture du recueil une nouvelle physionomie, assurée par la réunion de poèmes auparavant étrangers l'un à l'autre,

9. Réapparues récemment sur le marché du livre ancien, nous avons eu la chance d'en faire l'acquisition. Il constitue un document de première importance pour observer le poète au travail et la genèse de certains poèmes pour lesquels on ne dispose d'aucun élément. Voici la version primitive de la « Préface » : «Il existe des poèmes où le poète essaye sa chance; d'autres où le poète la prolonge. Rares sont les poèmes de chance. Parfois les plus rébarbatifs, ils coulent de la main comme l'ectoplasme de la bouche du médium. Le poète, endormi d'un œil, corrige, empêche la pâte de prendre et une forme confuse. / Ainsi naissent des mandragores : statues des profondeurs. Réunir cette sorte de poèmes, les retoucher, éliminer le reste, représente une tâche ingrate ; car le public aime reconnaître ; la connaissance le fatigue et rarement il approuve ce que le livre apporte de nouveau. » 


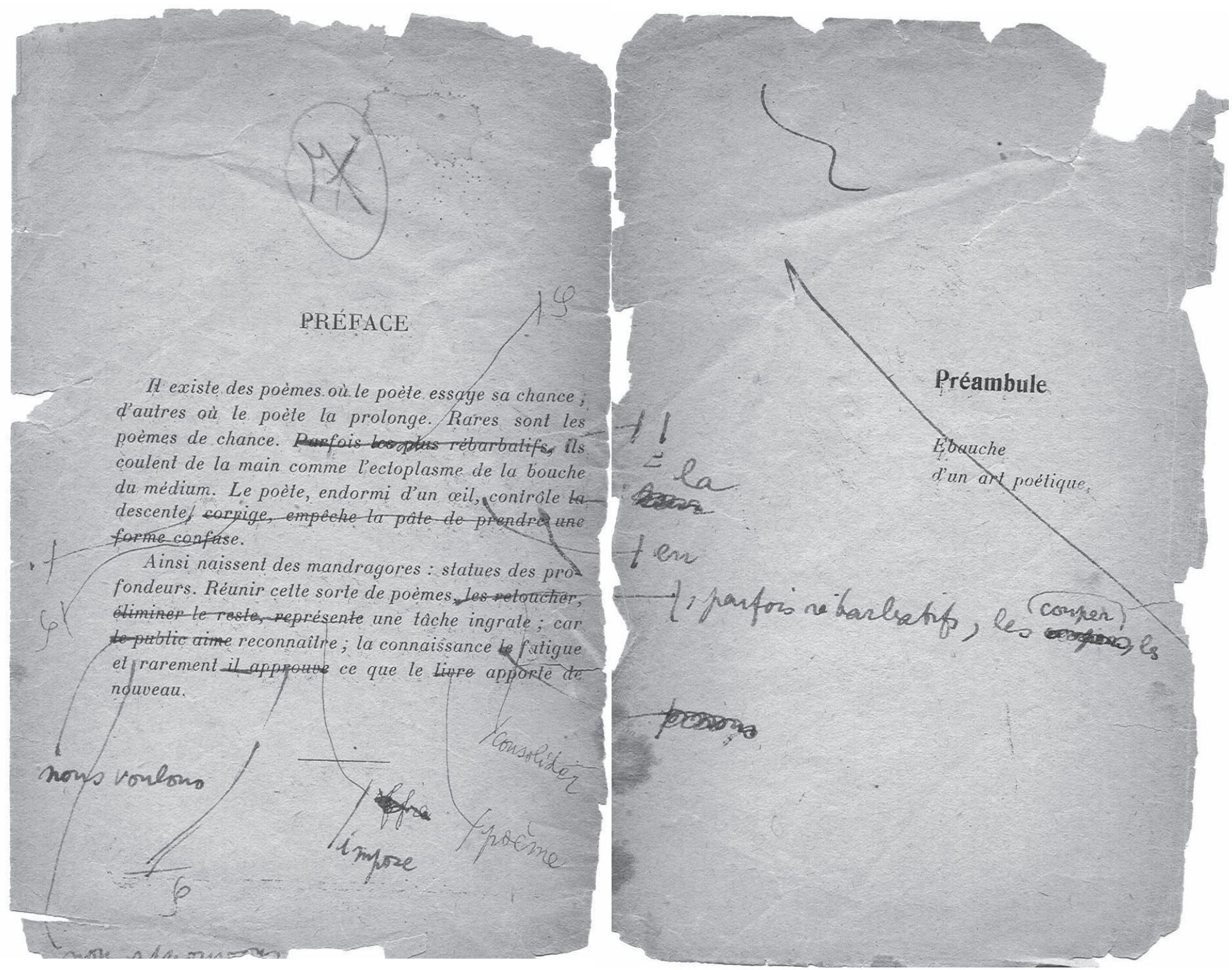

Fig. 1 : Jean Cocteau, « Préface » et « Préambule » à Morceaux choisis, épreuves corrigées (coll. particulière, (c) Comité Jean Cocteau) 
et l'observation d'une architecture qui fait alterner des jeux de motifs antagonistes (vie et mort, silence et bruit, jour et nuit, impuissance créatrice et génie inspiré, etc.) et de formes variées (vers et prose, pièces courtes et longues, vers isométriques et hétérométriques, etc.).

Détachés des circonstances qui les ont vu naître, les poèmes de Morceaux choisis ne gardent de leur première publication que le lien qui les unit aux autres poèmes parus dans un recueil commun. La temporalité et l'espace d'origine ont disparu ; le déplacement de certains des poèmes sélectionnés par Cocteau dans un nouvel espace de parution répond au désir du poète de prendre la mesure du temps et le recul nécessaire pour réévaluer ses pièces au sein de l'œuvre dont l'unité est questionnée. À l'occasion de ce transfert, Cocteau - on le verra - modifie la disposition des mots sur la page, l'ordre de succession des poèmes, voire la chronologie des recueils séparés, pour tenter l'aventure d'un nouvel équilibre. La sélection qu'il a opérée est soumise à des critères multiples, qui relèvent autant de la chronologie que de l'esthétique (sujets et formes). Le résultat produit offre l'exemple d'un nouvel ordre poétique - tendu vers l'unité - et d'une étape de l'histoire des textes - soumise à l'élaboration génétique en cours. Recomposition constituée à partir d'éléments disloqués, le florilège de Morceaux choisis correspond à la définition que F. Dumont donne de l'anthologie qui « est à la fois le signe de l'importance du recueil dans la diffusion de la poésie et le symptôme d'une mise à l'écart du recueil dans la lecture de la poésie10 ». Ce faisant, on observe un Cocteau auteur et lecteur de sa poésie, qui a retenu des pièces qui devaient paraître à ses yeux comme les plus représentatives de son œuvre entre 1916 et 1930. Or cette sélection qui se voit arrangée dans une dispositio nouvelle génère une seconde inventio du poème dont il convient à présent d'analyser les modalités.

\section{Modalités génétiques : \\ Cocteau lecteur et correcteur de ses œuvres}

Le regard neuf que Cocteau porte sur ses poésies antérieures a pour effet de renouveler l'invention thématique, la disposition des parties et l'élocution de détail. Bilan poétique, Morceaux choisis offre au poète l'occasion de redessiner la physionomie de sa phrase, l'espace graphique de son poème et le cadre spatial du livre qui résume tous les autres. À l'instar de tous les ouvrages de Cocteau, celui de 1932 a fait l'objet d'une attention suivie dont on peut retracer partiellement les étapes génétiques. Jusqu'à présent, on ne connaissait pour ce volume que l'histoire de la phase éditoriale11 constituée à partir des documents de genèse exogènes (manuscrits, éditions préoriginales, originales et rééditions de chaque recueil séparé de l'anthologie12). Nous sommes à présent en mesure d'éclairer la phase prééditoriale grâce au jeu des premières épreuves de Morceaux choisis, sur lesquelles s'observent les traces de nombreux remaniements opérés par Cocteau 13. On parvient ainsi à reconstituer l'histoire génétique de cette anthologie qui éclaire par ailleurs, singulièrement, la genèse individuelle des six livres qui la composent 14 . On dispose ainsi d'un dossier de genèse dont les phases se décomposent en quatre phases, depuis la rédaction de pièces individuelles jusqu'aux rééditions collectives augmentées :

[A. Phase prérédactionnelle : notes, fragments de rédaction, ébauches

B. Phase rédactionnelle : brouillons, versions successives, mises au net corrigées]

C. Phase prééditoriale : manuscrit définitif, placards/jeu d'épreuves corrigés

D. La phase éditoriale : préoriginale, édition originale, rééditions (Morceaux choisis), éditions postérieures et posthumes

10. Voir «Formes et fonctions de l'anthologie en poésie », dans Méthode!, op. cit., p. 33.

11. Nous suivons la terminologie de P.-M. de Biasi, résumée notamment dans La Génétique des textes, Paris, Nathan, 2000, p. 48 sq.

12. La table des matières de Morceaux choisis rappelle succinctement les livres mis à contribution : Le Cap de Bonne-Espérance, Discours du grand sommeil, Poésies (1920), Vocabulaire, Plain-chant et Opéra.

13. Ce jeu d'épreuves se présente sous la forme de vingt-trois placards in-12. L'ensemble, daté du 27 octobre 1931, est incomplet du dernier placard correspondant aux pages 166-173 du livre imprimé en juin 1932. Jauni et fragilisé par l'acidité, il présente parfois de nombreuses corrections autographes que nous analyserons plus loin.

14. Les éditeurs des Euvres poétiques complètes de Cocteau dans la « Bibliothèque de la Pléiade » retracent parfaitement la genèse complexe de chacun de ces recueils et fournissent des documents matériels abondants et précieux pour comprendre la méthode de travail de ce poète. 
Les deux premières phases et une partie de la quatrième concernent d'abord l'histoire des livres poétiques pris séparément, avant qu'ils ne fassent l'objet de la sélection par Cocteau en 1931-1932 (éléments indiqués en gras). Mais à partir de Morceaux choisis, leur genèse prend un tournant car les remaniements que Cocteau apporte sur ces poèmes antérieurs seront conservés pour leurs rééditions ultérieures. En somme, l'anthologie de Morceaux choisis constitue une étape opératoire et décisive dans l'évolution de l'œuvre poétique. On notera enfin que des éléments matériels manquent encore pour compléter la reconstitution de la préhistoire de l'anthologie : la copie manuscrite des poèmes choisis (ou la version imprimée de base), le jeu des secondes épreuves corrigées. De l'avant-texte à l'après-texte, la genèse de Morceaux choisis éclaire autant le passé que le devenir des livres édités séparément, et de l'anthologie de 1932 qui reste une expérience unique dans la carrière littéraire de Cocteau. Abondamment annotée sur les épreuves, l'anthologie voit son contenu et sa forme évoluer selon trois aspects : l'invention, la disposition et l'élocution des textes.

C'est naturellement dans le choix opéré des poèmes antérieurs que se construit le contenu ou l'invention du nouveau recueil. Choisir, c'est retenir mais aussi exclure. Dans cette optique, le geste de la sélection des poèmes est aussi important que celui de la rature portant sur les mots du poème. Comme le montre le jeu d'épreuves corrigées de Morceaux choisis, Cocteau a soigneusement médité le choix de ses pièces. Du Cap de BonneEspérance, il avait retenu d'abord le « Préambule » et «L'invitation à la mort»: sur les épreuves, il biffe la première pièce ou fragment d'un art poétique (voir fig. 1). Au Discours du grand sommeil, l'anthologie emprunte la moitié des pièces, celles qui sont marquées par une tonalité mortuaire15. Du volume copieux de Poésies, 1920, Cocteau n'a choisi que dix poèmes sur soixantedix, eux aussi marqués par l'expérience douloureuse de la guerre et la hantise de l'art ; c'est dire le choix drastique opéré ici par rapport à l'anthologie précédente parue en 1925 (Poésie 1916-1923)16. Autre sélection radicale, celle qui s'opère dans Vocabulaire : dans des proportions semblables à celles de Poésies, 1920, la section Vocabulaire reprend douze des soixante-cinq pièces qui la composaient en 192517. Enfin, des trois divisions de Plain-chant de 1923-1925, Cocteau n'a retenu que les deux dernières en 1932, éliminant la première chargée de présenter au lecteur sa poétique, et se recentrant sur l'expérience du vécu18.

Pour caractériser la sélection effectuée par Cocteau en 1931-1932, on peut dire qu'elle respecte l'ordre antérieur des livres individuels, qui sera inchangé jusqu'au bout, mais qu'elle procède à une sélection très réduite de leur contenu, variant d'une section à l'autre. Les suppressions opérées par rapport aux éditions originales séparées et à la collective de 1925 ne sont pas sans altérer la réception des poèmes choisis. La variété thématique se voit resserrée au profit de la densité et d'une plus forte homogénéité. La varietas du recueil collectif de 1925 contraste avec le principe d'économie de l'anthologie en 1932.

Aux changements quantitatifs qui allègent le recueil, Cocteau a compensé par l'ajout d'Opéra, un livre paru séparément en 1927 et qui fut mal accueilli par la critique. Celui-ci referme Morceaux choisis et procède également à des coupes sombres par rapport au massif original : de la soixantaine de pièces, il n'a gardé que la moitié. C'est proportionnellement plus que pour les livres précédents ; ce choix s'explique peut-être aussi par la forme des poèmes dont Cocteau retient surtout les pièces versifiées. Opéra constitue en tout cas la véritable nouveauté de l'anthologie de 1932. Ajouté in fine, à la suite des autres sections du recueil, Opéra possède un statut à part19. Alors que les rééditions ultérieures (1936 et 1947) du

15. Ce sont le « Prologue », une partie du « Discours du grand sommeil », «Malédiction au laurier », "Délivrance des âmes », « Désespoir du Nord », et «L'adieu aux fusiliers marins ».

16. Ces dix poèmes sont « Premières larmes », « Oceano roof », « Fête de Montmartre », « Batterie », « Pauvre Jean », «L'Ode à Picasso », « Gravité du cœur », « Le secret du bleu », « Louange de l'olivier » et « L'exécution ».

17. « Hôtel de France et de la poésie », « Ciel d'avril », « La mort de l'amiral », « Pièce de circonstance », « Mort d'un cygne », « Idole », « Dos d'ange », « À force de plaisirs... », « Angélus », « Les anges maladroits », « Rosier sauvage », et « M'entendez-vous ainsi ? ».

18. Sur la genèse de cette section, voir F. Demeulder et M. Dominicy, «Plain-chant : écriture et composition d'un recueil de Cocteau », dans Méthode!, op. cit., p. 141-156.

19. Sur cette section, voir la notice que lui consacre D. Gullentops dans l'édition des Euvres poétiques complètes, éd. citée, p. 1685-1692. 
volume Poésie 1916-1923 reprendront l'architecture d'origine, elles délaisseront Opéra. Seule l'édition des Poèmes (1916-1955), en 1956, accueillera certaines de ses pièces mais au prix d'une refonte complète de l'anthologie. En somme, dans Morceaux choisis, Cocteau a plus supprimé qu'il n'a ajouté, ce qui est paradoxal car il avait au contraire grossi ses volumes collectifs de Poésies précédents par l'apport de compositions récentes. L'anthologie de 1932 est donc bien le fruit d'un projet inverse qui, bien que s'inscrivant dans la tradition par l'ajout de poèmes récents (Opéra mais aussi « Le camarade » placé à la suite20), s'efforce de modifier la trame antérieure, connue du lecteur, pour en modifier le contenu. La diminution du nombre des poèmes imprimés (cent soixante en 1925 contre soixante-six en 1932) aboutit à la réalisation d'un recueil allégé rééquilibrant le rapport entre les parties et le tout.

Cet ordre de la sélection qui altère l'unité du recueil affecte aussi l'ordre de la disposition des pièces au sein du volume et parfois au cœur des pièces individuelles. Cocteau, en effet, s'ingénie à placer et à déplacer ses poèmes, à modifier la disposition des vers, la ponctuation de la syntaxe, en bref à modifier la composition graphique des poèmes sur la page. Ces remaniements autographes, qu'on observe sur le jeu d'épreuves corrigées de Morceaux choisis, témoignent d'une élaboration créatrice intense qui prouve l'importance que Cocteau accordait à ce nouveau livre. Loin d'être une simple réimpression de pièces connues, le recueil de 1932 questionne le bienfondé de l'ordre antérieur et oriente l'écriture poétique vers de nouvelles recherches.

Si l'on constate chez Cocteau l'existence de deux types d'ouvrages, selon le degré atteint d'unité dans le livre (Le Cap de Bonne-Espérance, Discours du grand sommeil) ou l'ouverture du recueil (Poésies 1917-1920)21, il faudrait ranger Morceaux choisis dans le second. Pourtant, le travail de redisposition effectué dans les poèmes et les sections conduit à réévaluer la fonction du recueil en livre.

Cette réorganisation spatiale, on en mesure les effets d'abord dans la répartition des poèmes en sections. Si Cocteau sélectionne ses pièces parmi ses livres antérieurs et les laisse dans leur cadre initial, en revanche il refond ponctuellement l'une ou l'autre des sections. C'est ainsi que « L'Ode à Picasso », publiée séparément en 1919 puis réunie à Poésie 1916-1923 (1925), est mise en valeur dans Morceaux choisis. Ce poème a sa propre page de titre et figure sous un titre distinct des Poésies, 1920 en table des matières. Cette nouvelle disposition n'est pas fortuite ; elle correspond chez Cocteau au désir de rendre un hommage vibrant au peintre qu'il vénère en ces années trente, et à une des vocations de Morceaux choisis qui place la poésie sous le signe de l'avant-garde esthétique et de la complémentarité des talents artistiques du poète, à la fois dessinateur et mélomane. De même, dans la section Opéra, autre section au titre générique associant les arts, « Musée secret » fait l'objet d'un traitement particulier : une page de titre individuelle l'isole comme subdivision dans le massif des poèmes d'Opéra, et souligne l'intérêt marqué pour ces sept poèmes, tous repris en 1932, où se lisent les visions d'un poète hanté par l'intrusion de figures ancestrales dans l'univers quotidien.

Affectant la configuration externe du livre, la réorganisation modifie aussi l'ordre des pièces dans chaque section. Entre 1925 et 1947, il ne change pas ; en 1932, pourtant, il évolue ponctuellement, provisoirement, comme si Cocteau cherchait à rééquilibrer les composantes des sections et à effriter les cloisons des parties pour faire dialoguer les poèmes entre eux. Ainsi, en éliminant sur les épreuves le «Préambule » du Cap de Bonne-Espérance, et en choisissant la seule «Invitation à la mort », Cocteau décide de ne plus expliciter les circonstances biographiques (sa rencontre avec l'aviateur Roland Garros) et littéraires (l'écriture d'une poésie d'avantgarde) de son projet, mais d'évoquer son baptême de l'air en compagnie de celui qu'il voit comme un héros. Cette « initiation » constitue une parabole de l'aventure poétique, faite d'un désir grisant d'élévation et menacée par la pesanteur qui conduit inéluctablement à la chute. Dans d'autres sections de Morceaux choisis, Cocteau a

20. Poème évoquant R. Roussel, tiré du film de Cocteau Le Sang d'un poète, qui fut présenté le 20 janvier 1932. Le poème est réuni à Opéra pour la première fois en 1932, et non en 1947 comme on l'a cru jusqu'ici (voir J. Cocteau, Euvres poétiques complètes, éd. citée, p. 1703). C'est dire si Morceaux choisis constitue bien un espace d'attente.

21. Voir les remarques de P. Caizergues dans la notice de Poésies (19171920), dans Euvres poétiques complètes, éd. citée, p. 1588. 
modifié l'ordre de ses poèmes. C'est le cas de Poésies (1920) où l'ordre des pièces sélectionnées s'écarte de celui de 1925 : «Premières larmes » (1), « Oceano roof » (6), «Fête de Montmartre » (34), «Batterie » (9), «Pauvre Jean » (22), «L'Ode à Picasso » (23), « Gravité du cœur » (61), « Le secret du bleu » (54), « Louange de l'olivier » (68), et « L'exécution» (36). Le choix opéré recentre le discours poétique autour du je biographique et littéraire et délimite un contenu grave entre les larmes du début et «L'exécution » qui évoque le motif ancien du front militaire. Vocabulaire (1922), recueil abondant, se voit réduit et réorganisé en 1932. " Hôtel de France et de la poésie » (1), réduit à un fragment, «Ciel d'avril » (9), «La mort de l'amiral » (4), « Pièce de circonstance » (6), « Mort d'un cygne » (17), « Idole » (18), « Dos d'ange » (31), « À force de plaisirs » (38), «Angelus » (46), « Les anges maladroits » (57), «Rosier sauvage » (53), et «M'entendez-vous ainsi » (62). Constitués en section, ces poèmes orientent le discours vers le motif de l'écriture même, l'inspiration, le sacrifice du génie, le sacré et la religion d'une vocation poétique qui interpelle finalement un public ingrat.

Cette redistribution des pièces dans chaque partie, qui distingue Morceaux choisis de Poésie (1916-1923), ne va pas de soi. Sur ses épreuves, Cocteau corrige, modifie, change d'avis sans cesse sur la place à assigner à ses poèmes. Soit qu'il voulût corriger une erreur du compositeur, soit qu'il désirât ébaucher une nouvelle alternance dans l'ordre des pièces, il ordonne sur ses épreuves de placer "Malédiction au laurier » après le douzième groupe de vers du "Prologue » de son Discours du grand sommeil. Il lui demande d'intervertir «Angélus » et «Les anges maladroits» (Vocabulaire), «Le théâtre grec » et «Nuit blanche» (Opéra). Il supprime également des poèmes comme "C'était déjà Noël sans raison », placé après « La nuit, l'Yser... » (Discours du grand sommeil), ou " Foire du Trône ", placé après « Hôtel de France... » (Vocabulaire).

Dans sa quête d'une architecture renouvelée et d'une disposition intrinsèque du recueil, Cocteau s'attache aussi à la formulation de chacun des poèmes. Si tous n'ont pas subi la marque de sa lime de manière égale, en revanche beaucoup ont fait l'objet de remaniements qui affectent la disposition comme l'élocution des vers. D'abord, on remarque sur les épreuves l'activité manuelle de Cocteau qui dispose et redispose ses vers sur la page comme un sculpteur, un peintre ou un musicien. Ainsi, la poésie du Cap de Bonne-Espérance devient une composition plastique. Souhaitant conférer à sa ligne poétique une forme aérienne, emblématique de son sujet, Cocteau désarticule le vers et le dispose sur la page comme Mallarmé et Apollinaire lui en avaient offert l'exemple 22 . Il invite ainsi à une lecture graphique du poème qui est distribué dans la spatialité de la page. À l'occasion de sa réimpression de 1932, Cocteau lui fait subir de nouveaux changements qu'on peut résumer à deux principes : économie de l'espace et simplification typographique. Cette tendance, que l'on observait entre l'impression princeps de 1918 et la réédition de 1925, s'accroît ici. Cocteau ajoute des capitales en début de vers pour mieux distinguer les mouvements ou segments de son poème, des signes de ponctuation pour clarifier l'expression ou l'infléchir dans une voie nouvelle, découpe exceptionnellement un vers et sa syntaxe pour créer un effet précis ${ }^{23}$. Le Discours du grand sommeil, qui relève d'une esthétique très voisine, procède à des corrections de même nature.

Au cours de son travail de révision, qui a débordé largement l'objectif de départ, à savoir la correction d'épreuves, Cocteau a apporté de nombreux autres éléments qui ont transformé son texte primitif. Outre les déplacements des vers ou segments de vers sur la page, il lui arrive d'alléger ses pièces en cours d'impression, un aspect du poète correcteur de ses œuvres qui correspond à ses habitudes de travail manuscrit. Ainsi, il coupe sur les épreuves cinq séquences de vers du Discours du grand sommeil24, ellipse volontaire qui accélère l'écoulement de la narration. Dans « Le secret du bleu », il a éliminé le deuxième paragraphe 25 avant même la composition des premières épreuves, tout comme six strophes de «Prière mutilée ». Dans «Hôtel de France », il a supprimé deux longs passages du début du poème 26 . Ces

22. Voir I. Chol, « La poésie spatialisée depuis Mallarmé », Poétique, $\mathrm{n}^{\circ} 158$, avril 2009, p. 231-247.

23. Voir l'édition de 1925, Paris, Gallimard, p. 13, 15, 16, 19, et 20

24. Voir l'édition des Euvres poétiques complètes, éd. citée, p. 437-438.

25. Ibid., p. 220.

26. Ibid., p. 293-294. 
allégements sont peut-être dictés par la finalité même du livre de Morceaux choisis, celle de la sélection qui oblige à éliminer des pièces, et à couper les plus longues d'entre elles. Mais ici, Cocteau expérimente l'expressivité de ses compositions, en mesure l'équilibre, la clarté sémantique, la justesse sonore. C'est pourquoi certains remaniements opérés en 1932 seront conservés dans les éditions décisives de 1947 et 1956 de ses poésies.

Le travail de révision lui offre aussi l'occasion de se remémorer les circonstances de composition de sa poésie. Reprenant ses livres les plus anciens et qui ont marqué fortement son existence durant la guerre (Le Cap de Bonne-Espérance, Discours du grand sommeil), Cocteau réévalue ses vers à l'aune de sa mémoire et de la place qu'occupe sa poésie dans la totalité de sa production littéraire conçue et répartie comme une vaste Poésie («Poésie », «Poésie de roman », «Poésie de théâtre », etc.). Il lui arrive alors de disséminer dans ses épreuves des marques de régie qui éclairent son histoire personnelle. Ainsi, le poème «Souvenir des souvenirs » portait-il d'abord le titre manuscrit « Toulon », écho biographique qui précise le lieu de composition au moment où le poète séjournait sur la Côte d'Azur (Villefranche, 1925). De même, lors de la correction d'épreuves, Cocteau ajoute un astérisque au poème «Le train musical » (*Air : Dix filles dans un pré), note qui oriente soit vers l'origine, soit vers la finalité de ce poème. Enfin, « L'Ode à Picasso » comporte à partir de 1932 la mention «(Version définitive) », note de régie reprise par la suite mais démentie par la forme du poème.

Car Cocteau, soucieux du perfectionnement incessant de son œuvre, retouche ses textes dans les moindres détails. Nous ne pouvons offrir - dans le cadre étroit de cet article - un recensement exhaustif de toutes les variantes relevées dans les textes de Morceaux choisis. Leur nombre et leur nature nécessiteraient une minutieuse analyse à elle seule. Mais pour donner un aperçu des transformations que Cocteau fait subir à ses textes, prenons l'exemple significatif de «L'Ode à Picasso », un texte dont P. Caizergues a remarquablement rappelé les circonstances de publication ${ }^{27}$. Connue par plusieurs manuscrits successifs et des éditions multiples, signe de l'attachement que lui portait le poète, « L'Ode à Picasso » parut en 1917 en plaquette puis fut réunie au volume collectif de Poésie 1916-1923, en 1925, avec pour soustitre «Version nouvelle ». Jusqu'ici, la critique a cru bon de n'en examiner que les versions de 1925, déjà citée, et de 1947 (t. III des CEuvres complètes chez Marguerat), car elles présentent entre elles de notables divergences. Or bon nombre des remaniements enregistrés en 1947 apparaissent déjà dans la version des Morceaux choisis, sans compter que d'autres variantes restent exclusives à cette édition-ci. D'où l'intérêt, semble-t-il, d'en offrir un relevé explicatif.

Le premier volet de ce diptyque, «L'homme assis », se voit allégé des cinq vers initiaux en 1932, suppression entérinée par l'édition de 1947 :

$$
\begin{aligned}
& \text { Souvenir de Montparnasse. } \\
& \text { «O ma jolie» } \\
& \text { Les places d'ombre. } \\
& \text { Fume sa pipe. } \\
& \text { Quittons l'Espagne. }
\end{aligned}
$$

Supprimant cette image mémorielle de l'artiste au travail, et de son passé, Cocteau ne retient plus que la suite qui s'attache à louer «le dompteur des muses », motif déployé dans le second volet. Celui-ci aussi subit plusieurs métamorphoses de 1925 à 1947, et là encore la version de 1932 constitue une étape importante de ce cheminement. Si depuis 1925, le texte a été totalement remodelé dans sa forme plastique pour être transposé sur le mode poétique et prendre une physionomie typographique qui demeurera par la suite, le détail des lignes varie, se précise, par le travail sur l'élocution. Cocteau montre sur ses épreuves une hésitation pour désigner une attitude (« l'œil du maître/ la replace » en 1925 et 1947 , puis « la repousse » en 1932), mais améliore la caractérisation (« son pouce montre un éventail » en 1925, «étale un éventail » en 1932 et 1947). Il parvient à atténuer une image par une périphrase ( la petite Erato trépigne/d'être complètement chauve » en 1925, «d'être belle sans cheveux » en 1932-1947). Un remaniement plus

27. Voir la notice qu'il lui consacre dans ibid., p. 1577-1582. 
important de l'énonciation transforme parfois une description réaliste en un petit tableau animé :
1925

Le fauteuil chante Ma Paloma.

Les quatre cloisons

s'approchent,

et les objets

suivent Orphée

jusqu'où il veut.

Dame de carreau

BASS

losange rouge,

Clio du zinc,

Calliope téléphone les faits-divers et Uranie allume

qui fardent les marronniers par-dessous. les becs de gaz
1932-1947

Écoutant ta guitare fée, les objets te suivent Orphée jusqu'à la forme que tu veux.

Clio du zinc, Calliope téléphone les faits-divers et Uranie allume les becs de gaz $^{28}$ qui fardent les marronniers pardessous.
La suite du texte présente d'autres variantes par rapport à la version de 1925 : variantes lexicales plus évocatoires, suppressions de mots, répétitions de mots à fonction eurythmique, découpage des vers accélérant le rythme de la phrase ${ }^{29}$, autant d'écarts qui enregistrent la réaction critique de Cocteau devant son poème et qui soulignent son effort pour en accroître la clarté musicale et la netteté visuelle.

La fréquence des variantes relevées et l'ampleur des transformations apportées aux poèmes de Morceaux choisis ne sont point le fruit d'une attitude capricieuse ou d'une réaction fortuite. Elles révèlent plutôt l'affirmation d'un écrivain qui souhaite reprendre le contrôle de son texte et le faire évoluer dans le sens de sa sensibilité actuelle. Dans le processus de sélection des poèmes, le choix des pièces écartées est aussi riche d'enseignements que celui des pièces choisies. De même, la liste des transpositions et des corrections apportées aux textes (ajouts, suppressions, permutations, substitution de variantes, etc.), dont nous n'avons qu'ébauché l'analyse, souligne l'intervention déterminée du poète qui entend reconfigurer son recueil.

\section{Vers une nouvelle orchestration : économie et sens du recueil}

Si le lecteur de 1932 croit déjà savoir ce qu'il va lire lorsqu'il ouvre la première page de Morceaux choisis - ou « reconnaître » son contenu, malgré la mise en garde de Cocteau dans sa «préface »-, il ne peut que s'étonner de découvrir au fil des pages le caractère «nouveau » de sa physionomie. En fait, Cocteau poursuit dans ce recueil d'attente sa recherche vers une plus grande expressivité à partir d'une sélection de pièces provenant de recueils publiés antérieurement. Son approche des textes ne répond point totalement au principe d'expansion (car seul Opéra vient se joindre à l'édition collective de Poésies) mais à celui du recentrement, une exploration du dedans du recueil pour corriger chacun des poèmes, délimiter le cadre des livres séparés devenus sections du recueil, et équilibrer les sections entre elles en tissant un réseau complexe de relations, de correspondances et d'asymétries entre poèmes et sections du livre. Morceaux choisis offre ainsi l'exemple d'une expérimentation sur les thèmes et les formes de prédilection de Jean Cocteau qui aboutit à une construction symphonique. À partir du thème central de la construction du langage, Cocteau développe divers motifs qui relèvent de sa mythologie personnelle sur une ligne rythmique majeure qui joue des effets multiples et variés de la scansion littéraire. Le résultat produit par l'écriture se caractérise par une très grande unité qui ménage une place à la liberté formelle, ou varietas.

Cocteau renouvelle la facture de ses Poésies en rebaptisant son recueil Morceaux choisis et en lui accolant un sous-titre, « Poèmes ». L'inverse n'eût-il

28. Les épreuves donnaient d'abord « la rampe verte », variante ensuite corrigée de la main de Cocteau et retenue dans la version éditée.

29. Notons ainsi les corrections suivantes : « Les tambours bleus vous font taire,/reines bavardes » (1925) devient «Alors les tambours de Santerre/vous font taire, reines bavardes » (1932-1947); « elle titube et oublie » (1925) contre « elle titube et elle oublie » (1932-1947); « Il vous séquestre » (1925) mais «Il vous épouse. Il vous séquestre » (19321947) ; l'image « où les anges écrivent leurs lettres/autour des arbres de Noël » (1925) s'assombrit avec « où la mort écrivait ses lettres sous un lustre de Noël » (1932-1947) ; Cocteau avait d'abord noté à la main sur ses épreuves : « où chacune écrivait ses lettres/sous le givre de Noël ». 
pas été plus adéquat pour intituler le nouveau livre? L'inversion paradoxale du titre et du sous-titre - peu conforme aux habitudes éditoriales qui mettent en avant le genre littéraire puis désignent son contenu - suggère au lecteur qu'il a affaire à un nouveau livre au titre inédit. Et c'est bien l'effet que Cocteau cherche à produire sur son lecteur. Celui-ci peut ensuite constater que, si la matière du livre lui est connue, sa manière lui apparaît sous un jour nouveau. Le poète ne bouleverse pas de fond en comble l'ordre de sa poésie établi dans Poésie 1917-1923 (1925). Il en garde l'ossature principale et reprend l'ordre chronologique de parution de chacune des parties qui ne suit point l'ordre de composition des pièces. C'est dire si Cocteau privilégie ici la prérogative de l'art sur la vie, même si l'histoire personnelle du poète est sous-jacente dans chacun de ses livres.

En fait, Cocteau juxtapose ou plutôt entremêle dans Morceaux choisis plusieurs perspectives temporelles. À l'ordre successif des livres dans le recueil, correspondant à la chronologie de parution, s'ajoute une ligne autobiographique, un ordre sentimental par lequel l'Histoire est racontée par le je. Cette conception d'une poésie personnelle, lyrique, n'est pas neuve chez Cocteau ; ce qui change dans Morceaux choisis, c'est que le resserrement des parties du livre s'accompagne d'une réduction d'épisodes biographiques, qui prennent la forme de moments et de rencontres exceptionnels. Ces poèmes choisis par le poète concentrent les étapes ou temps forts de sa vie, de 1915 à 1931, et sont rangés selon plusieurs périodes organisées dans des sections emblématiques de poèmes : Le Cap de Bonne-Espérance et le Discours du grand sommeil rassemblent l'expérience de la guerre ; L'Ode à Picasso représente la découverte de la peinture et de son union à la poésie ; Vocabulaire, Plain-chant et Opéra placent la révolution poétique sous le signe de la peinture et des autres arts. À chaque fois, il s'agit pour Cocteau d'explorer les formes de la poésie et de questionner le statut, le rôle et les fonctions du langage.

À la ligne sentimentale discrète, dont les poèmes égrènent les confidences d'un bout à l'autre du livre, s'associe un ordre esthétique, à la fois plastique et musical. La succession des parties de Morceaux choisis simule l'expansion du lyrisme chez Cocteau, c'est-à-dire, selon la définition de Valéry, « le développement d'une exclamation 30 ». On ne peut qu'être frappé par l'évolution de l'expression poétique de 1916 à 1932 : d'un discours poétique éclaté ou de sa désorganisation volontaire, qui éparpille les mots sur la page selon les prérogatives de l'œil (Le Cap de Bonne-Espérance), la poésie de Cocteau a posé les éléments d'un nouveau langage poétique s'appuyant sur la définition d'un Vocabulaire (1922) et d'une oralité liturgique (Plain-chant, 1923). L'apparition d'Opéra dans le recueil collectif de ses poésies semble conclure cette aventure du langage et affirmer l'aboutissement d'une poésie orchestrale, sensible aux variations du langage poétique qui réconcilie les vers et la prose.

La réorchestration évolutive chez Cocteau conditionne d'abord les thèmes abordés dès 1914-1916. Dans Morceaux choisis, la sélection opérée à partir des recueils antérieurs consolide une vision poétique grave, marquée par la guerre et la mort. Ainsi, du Cap de Bonne-Espérance, Cocteau n'a finalement gardé que «L'invitation à la mort ». Dans le Discours du grand sommeil, synonyme de la mort, il a retenu les séquences dominées par la guerre et les images qui hantent sa mémoire. La gravité des poèmes suivants ne semble offrir à la conscience du poète aucun apaisement, sinon le désir d'en finir avec une époque de fraternité douloureuse («L'adieu aux fusiliers marins »). De Poésies, 1920, où s'observait le regain d'une activité poétique soutenue par le retour à la vie civile, Cocteau a reproduit des poèmes où la tristesse se fait entendre en sourdine («Premières larmes », «Pauvre Jean », «Gravité du cœur », «L'exécution »), malgré la joie éprouvée devant le spectacle de l'art («L'Ode à Picasso »). Dans Vocabulaire, cette tendance se confirme et se précise : l'exercice vivifiant de la poésie qui doit compenser la conscience souffrante du poète ne parvient guère à exorciser les démons du passé et ceux du présent. L'alphabet poétique dont se dote le poète lui permet de se construire une nouvelle identité et une voix qui implore autant les anges salvateurs qu'elle se lamente sur la «Mort d'un cygne ». Le dernier poème

30. Dans Tel quel. Cité par J.-M. Maulpoix, La Voix d'Orphée. Essai sur le lyrisme, Paris, José Corti, 1989, p. 71 sq. 
de cette section («M'entendez-vous ainsi ? »), en 1932, renouvelle l'appel lancé à l'inspiration dans un dialogue du poète avec un public français sourd à ses requêtes. Plain-chant constitue alors un tournant dans le déroulement thématique de Morceaux choisis. « Je veux tout oublier » s'exclame Cocteau à l'ouverture de cette section qui débute par le deuxième volet. Renouant avec une poésie versifiée, Cocteau s'interroge sur les aléas de l'inspiration et définit un nouvel art poétique, sous la tutelle des Muses, dans lequel il perçoit un nouveau départ existentiel :

Si ma façon de chant n'est pas ici la même,

Hélas, je n'y peux rien.

Je suis toujours en mal d'attendre le poème,

Et prends ce qui me vient.

[...]

Or moi j'ai secondé si bien leur force brute,

Travaillé tant et tant,

Que si je dois mourir la prochaine minute,

Je peux mourir content 31 .

L'espoir envisagé ici, qui conclut Plain-chant dans le recueil collectif de 1932, semble amener logiquement la dernière section, Opéra, dont les poèmes retenus occupent soixante des cent soixante pages que comporte Morceaux choisis. C'est dire si Cocteau a souhaité souligner le renouvellement de son inspiration et inaugurer un nouveau départ personnel en poésie. La sélection opérée par Cocteau présente un autoportrait, le résumé de son histoire depuis l'enfance jusqu'à l'âge adulte marqué par ses rencontres et des disparitions («L'Ange Heurtebise », dont la forme disloquée exprime la rupture amoureuse). Puis, avec «Musée secret », Cocteau poursuit son interrogation sur les mystères du langage et de l'art. C'est dans les lieux et les formes antiques surgissant dans son quotidien, et dans l'activité du sommeil et du rêve, que le poète cherche à capter les signes de l'invisible. Musée, théâtre, cinéma, opéra, tels sont les lieux fréquentés par un poète convaincu de leur nécessaire union et qu'il cherche à meubler par ses essais artistiques du moment.

Au bout du voyage que ponctue chacune des sections poétiques de Morceaux choisis, le lecteur a parcouru l'itinéraire personnel de Cocteau et peut percevoir l'évolution d'un poète qui est passé de la tragédie du passé à la comédie du présent. La création sert de fil conducteur mais elle est intériorisée, narrativisée tout au long du livre, Cocteau cherchant à effacer les marques les plus visibles de sa biographie. Du coup, son histoire personnelle se voit transportée dans une vision du monde qui transpose sur le mode poétique l'expérience du vécu. Les drames personnels (amoureux, physiques) prennent la dimension d'une épopée universelle grâce à un discours poétique qui s'élève au lyrisme.

C'est là sans doute qu'apparaît avec le plus de clarté la démarche de Cocteau dans Morceaux choisis. L'instance d'énonciation ne cesse de varier et subsume le je individuel en nous. Le je est l'écho d'autres je, doubles de l'homme, du locuteur et du poète. Cocteau se contemple, s'observe à distance, et interpelle d'autres voix auxquelles il donne parfois une place dans ses poèmes. Sa poésie, monologique et dialogique (correspondant avec les Muses, l'Ange), s'ouvre à la polyphonie.

Faut-il dès lors s'étonner si Cocteau expérimente autant de formes expressives ? Si sa poésie, consommatrice d'images, doit tant aux arts du visible, elle s'impose surtout par sa capacité à générer une multitude de modèles rythmiques. Que d'écart entre la discontinuité et le rejet de la rhétorique dans Le Cap de Bonne-Espérance et l'alternance de formes poétiques variées (en vers et en prose, en vers réguliers ou libres) déployées dans Opéra ! Il semble, là encore, que Cocteau ait minutieusement élaboré à la fois une trajectoire des formes rythmiques et un équilibre des formes d'une section à l'autre de Morceaux choisis. Sur l'axe linéaire, on constate une évolution progressive depuis la dislocation du langage (Le Cap de BonneEspérance), le passage à la maîtrise d'une poésie libre mais séquentielle (Discours du grand sommeil), culminant dans Vocabulaire, qui sert de transition entre une esthétique de l'avant-garde et une poétique classique, symptomatique de Plain-chant, qui est construit sur le seul quatrain hétérométrique (alternant alexandrins et hexasyllabes). Toutes ces expérimentations sur le vers et la strophe semblent converger finalement dans Opéra qui, réunissant des pièces en vers libres et en strophes, en vers

31. Morceaux choisis, éd. citée, p. 110 et 112. 
et en prose, effectue la synthèse des rythmes poétiques antérieurs. Dernier volet de Morceaux choisis, Opéra s'ouvre à tous les sujets, toutes les formes littéraires mais encore à tous les arts (musique, peinture, architecture et cinéma). Il constitue comme l'acmè emblématique du recueil de 1932, dont le rythme va crescendo.

Comme on le voit, Morceaux choisis porte mal son titre. Loin de n'être qu'un recueil de poèmes épars sélectionnés au gré de l'écrivain ou de son éditeur, ce nouveau livre se situe à un tournant de la carrière poétique de Cocteau. Euvre bilan, qui autorise le poète à reconfigurer l'assemblage des parties en une unité sans cesse recherchée, il constitue aussi une étape pour intégrer les compositions du passé dans une écriture tendue vers l'avenir. Pause réflexive, autocritique, correspondant à une période où Cocteau traverse une crise existentielle grave dont il tente de se libérer par l'écriture, le livre de 1932, fort de sa structuration antérieure qui évolue soudain, vise à l'élaboration et à l'expérimentation d'une mythologie et de formes modernes. Espace du repli, le livre présente des pièces remodelées, amputées ou augmentées, qui y ont trouvé refuge. Dans ce cadre provisoire, elles entament de nouvelles relations entre elles qui préfigurent la physionomie de l'édition suivante. Morceaux choisis offre ainsi l'exemple parfait d'une œuvre poétique en devenir et s'apparente au méandre d'une ligne tortueuse qui vise à l'infini32. Réédité à part en 1945 (Lausanne, H. Keyser), ce livre montre assez l'estime que lui portait Cocteau.

32. C'est en ces mots que Cocteau caractérisait l'apparent vagabondage poétique de Paul Valéry dans un article de la revue Fontaine (été 1945), repris dans les Cahiers Jean Cocteau, n 9, 1981, p. 163. 
François Rouget est professeur de littérature française à Queen's University (Canada). Spécialiste de la Renaissance, il a publié plusieurs ouvrages et articles sur la poésie de Clément Marot à Agrippa d'Aubigné, notamment sur le genre de l'ode (L'Apothéose d'Orphée, Droz, 1994 ; L'Arc et la lyre, SEDES, 2001) et plusieurs éditions critiques (O. de Magny, P. de Tyard, Album de vers de M. de Valois). Ses travaux actuels s'inscrivent dans la génétique textuelle de l'écriture chez P. de Ronsard (Ronsard et le livre, I, Droz, 2010). Il a par ailleurs fait paraître plusieurs études sur les manuscrits de J. Cocteau (en 2006 et 2008).

François Rouget, frouget30@gmail.com

Cocteau et la poétique du déplacement

La présente étude explore les modalités des déplacements opérés par Jean Cocteau dans Morceaux choisis, auto-anthologie, album ou florilège de poèmes composés et publiés de 1916 à 1927. À partir de l'enquête génétique de ce recueil, qui s'appuie sur l'étude des éditions séparées et collectives antérieures des poèmes qui le composent, mais aussi sur l'examen des épreuves corrigées de sa main qui ont été récemment retrouvées, il s'agit de s'interroger sur le statut de ce livre délaissé de la critique, sur les objectifs de l'écrivain et sur les effets esthétiques (plastiques, musicaux) produits par le déplacement et le nouvel agencement des poèmes. On y voit Cocteau composer un nouveau livre pour reconfigurer ses recueils antérieurs et ébaucher une unité provisoire de ses poésies qui furent louées ou détestées de son public. Au moment où il tente de conjurer ses démons (l'attrait de l'opium, une sexualité contrariée, l'angoisse de la mort), Cocteau procède à un bilan personnel et poétique des vingt années précédentes, et entend refonder les bases de sa vision poétique qui doivent lui permettre de dépasser ses échecs.

The present essay explores the modalities of displacement effected by Jean Cocteau in Morceaux choisis, an auto-anthology, an album or selection of poems composed and published between 1916 and 1927. With a genetic investigation of this volume, based on the study of previous separate and collective editions of its poems as well on the examination of the recently discovered corrected proofs, we examine the status of this book neglected by critics, and the esthetic effects (plastic, musical) produced by the displacement and the new arrangement of the poems. In this way we see Cocteau composing a new book in order to rearrange his previous anthologies and develop a temporary unity of his poems that had been alternately praised or condemned by his readers. While struggling to ward off his devils (the attraction of opium, a frustrated sexuality, the fear of death), Cocteau undertook an assessment of his life and of his poems of the previous twenty years, wishing to reestablish the foundations of his poetical vision which would allow him to overcome his failures.

D ie vorliegendeStudie setzt sichmit den Modalitäten der Verschiebungen auseinander, die Jean Cocteau in Morceaux choisis - Auto-Anthologie, Album und Florilegium von Gedichten, die zwischen 1916 und 1927 geschrieben und veröffentlicht wurden - vornimmt. Ausgehend von der textgenetischen Untersuchung dieses Bandes, die sich sowohl auf die vorausgegangenen Einzel- und Sammelpublikationen der darin enthaltenen Gedichte stützt als auch auf die von der Hand des Autors annotierten Korrekturfahnen, die man kürzlich wiedergefunden hat, geht es darum, nach dem Status dieses von der Kritik vernachlässigten Buches zu fragen sowie nach den Zielen des Schriftstellers und den ästhetischen (bildkünstlerische, musikalische) Effekten, die durch die Verschiebung und die Neuanordnung der Gedichte hervorgerufen werden. Cocteau stellt hier ein neues Buch zusammen, in dem er seine früheren Sammelschriften neu konfiguriert und eine provisorische Einheit seiner Gedichte entwirft, die von seinem Publikum gelobt oder verabscheut wurden. In dem Moment, in dem er versucht, seine Dämonen zu beschwören (die Anziehung durch das Opium, eine als problematisch empfundene Sexualität, die Angst vor dem Tod), zieht Cocteau eine persönliche und poetische Bilanz der zwanzig vorausgegangenen Jahre, mit der Absicht, das Fundament seiner poetischen Vision zu erneuern, um seine Misserfolge hinter sich zu lassen.
El presente estudio explora las modalidades de los desplazamientos efectuados por Jean Cocteau en Morceaux choisis, auto-antología, álbum o florilegio de poemas compuestos y publicados entre 1916 y 1927. A partir de la investigación genética de esta recopilación, que se basa en el estudio de las ediciones separadas y colectivas anteriores de los poemas que la componen, y también en el examen de las galeradas con correcciones autógrafas descubiertas recientemente, es posible interrogarse sobre la naturaleza de ese libro olvidado por la crítica, los objetivos del escritor y los efectos estéticos (plásticos, musicales) producidos por el desplazamiento y la nueva disposición de los poemas. Vemos en él a Cocteau componiendo un nuevo libro para reconfigurar sus recopilaciones anteriores y esbozar una unidad provisoria de sus poesías, que fueron alabadas o detestadas por su público. En el momento en que intenta conjurar sus demonios (la atracción del opio, una sexualidad contrariada, la angustia de la muerte), Cocteau efectúa un balance personal y poético de los veintes años precedentes, y trata de reestablecer las bases de su visión poética, que le permitan sobrepasar sus fracasos.

$\mathbf{O}$ presente estudo explora as modalidades de deslocação operadas por Jean Cocteau em Morceaux choisis, auto-antologia, álbum ou florilégio de poemas compostos e publicados de 1916 a 1927. A partir do inquérito genético desta colectânea, que se apoia no estudo das edições individuais e colectivas anteriores dos mesmos poemas, mas também no exame das provas corrigidas pela sua mão, recentemente reencontradas, pode questionar-se o estatuto deste livro esquecido pela crítica, os objectivos do escritor e os efeitos estéticos (plásticos, musicais) produzidos pela deslocação e a nova disposição dos poemas. Cocteau é visto a compor um novo livro para reconfigurar as colectâneas anteriores e a esboçar uma unidade provisória das poesias que foram elogiadas ou odiadas pelo público. Quando tenta conjurar os seus demónios (atracção do ópio, sexualidade contrariada, angústia da morte), Cocteau procede a um balanço pessoal e poético dos vinte anos precedentes, e procura refundar as bases da sua visão poética de forma a transcender os seus malogros.

Questo studio esplora le modalità delle dislocazioni operate da Jean Cocteau in Morceaux choisis, auto-antologia, album o florilegio di poesie composte e pubblicate dal 1916 al 1927. A partire dall'indagine genetica di questa raccolta, che si basa sullo studio delle edizioni separate e delle raccolte precedenti delle poesie che la compongono, ma anche sull'esame delle bozze corrette dall'autore, che sono state recentemente ritrovate, ci si interroga sullo statuto di questo libro trascurato dalla critica, sugli obiettivi dello scrittore e sugli effetti estetici (plastici e musicali) prodotti dallo spostamento e dalla nuova disposizione delle poesie. Si segue Cocteau che compone un nuovo libro per riconfigurare le sue raccolte precedenti e progetta un'unità provvisoria delle sue poesie che erano state lodate o detestate dal suo pubblico. Nel momento in cui egli tenta di scacciare i suoi demoni (l'oppio, una sessualità impedita, l'angoscia della morte), Cocteau offre un bilancio personale e poetico dei vent' anni precedenti, e intende rifondare le basi della sua visione poetica che gli devono permettere di superare le sue sconfitte. 